\title{
AVALIAÇÃO FUNCIONAL DOS PACIENTES SUBMETIDOS AO DESBRIDAMENTO ARTROSCÓPICO PARA TRATAMENTO DAS RUPTURAS EXTENSAS E IRREPARÁVEIS DO MANGUITO ROTADOR
}

\author{
FUNCTIONAL EVALUATION OF PATIENTS WHO HAVE UNDERGONE ARTHROSCOPIC \\ DEBRIDEMENT TO TREAT MASSIVE AND IRREPARABLE TEARS OF THE ROTATOR CUFF
}

Marco Antônio de Castro Veado', Alessandro Ulhôa Rodrigues²

\section{RESUMO}

Objetivo: Avaliar os resultados dos pacientes submetidos ao desbridamento artroscópico das lesões extensas e irreparáveis do manguito rotador. Métodos: Foram operados 27 pacientes no período de 2003 a 2007, sendo avaliados 22 desses. O procedimento cirúrgico consistiu de desbridamento artroscópico do coto dos tendões envolvidos, bursectomia, remoção do osteófito acromial e, eventualmente, tenotomia do bíceps e tuberoplastia. Resultados: No pré-operatório todos apresentavam envolvimento dos tendões do supra e infraespinal. Na avaliação pós-operatória, 14 pacientes estavam com o redondo menor íntegro e três com ruptura parcial do subescapular. Houve melhora dos critérios da UCLA de 15 no pré-operatório para 31 no pós. Não houve melhora de força muscular, porém ocorreu redução da dor. Conclusão: Desbridamento artroscópico é um procedimento indicado para pacientes idosos com ruptura irreparável do manguito rotador, que tenham boa $\mathrm{ADM}$, baixa demanda funcional e com o principal objetivo de reduzir a dor.

Descritores - Desbridamento; Artroscopia; Bainha Rotadora; Bursite

\section{ABSTRACT}

Objective: To evaluate the results in patients who have undergone arthroscopic debridement of massive and irreparable injury of the rotator cuff. Methods: 27 patients were operated in the period from 2003 to 2007, during which 22 of them were evaluated. The procedure used consisted of arthroscopic debridement of the related tendons of the residual limb, bursectomy, acromial osteophyte removal, and eventually, biceps tenotomy and tuberoplasty. Results: All patients showed involvement of the supraspinatus and infraspinatus tendons in the preoperative stage. In the postoperative evaluation, 14 patients had an teres minor muscle, and 3 had partial tears of the subscapularis tendon. There was an improvement in the UCLA criteria from 15 preoperatively to 31 postoperatively. There was no improvement in muscular strength, but there was a reduction in the pain. Conclusion: Arthroscopic debridement is a recommended procedure for elderly people with irreparable rotator cuff rupture, good ROM, low functional demand, and whose main objective is to diminish pain.

Keywords - Debridement; Arthroscopy; Rotator Cuff; Bursitis

com uma lesão extensa e irreparável do MR.

Lesões extensas são classificadas como aquelas maiores do que $5 \mathrm{~cm}$ e com envolvimento de pelo menos dois tendões ${ }^{(1)}$ e irreparáveis quando não podem ser reparadas naquele ato cirúrgico, seja pela grande retração dos cotos ou pela qualidade ruim dos tendões ${ }^{(2)}$.

1 - Professor da Faculdade de Ciências Médicas de Minas Gerais; Cirurgião do Grupo de Ombro e Cotovelo do Hospital Mater Dei e do Hospital Israel Pinheiro de Minas Gerais (IPSEMG).

2 - Cirurgião do Grupo de Ombro e Cotovelo do Hospital Mater Dei - Belo Horizonte, MG.

Trabalho realizado no Hospital Mater Dei e IPSEMG - Belo Horizonte, MG.

Correspondência: Avenida Barbacena, 1.018/806, Santo Agostinho - 30190-131 - Belo Horizonte, MG. E-mail: alessandroulhoa@superig.com.br 
A literatura oferece várias opções para o tratamento dessas lesões, como tratamento conservador com analgésicos, anti-inflamatórios não esteroides, infiltrações com corticosteroide, fisioterapia para fortalecimento do deltoide anterior, exercícios ativos e $\operatorname{assistidos}^{(3-5)}$. As opções cirúrgicas incluem desbridamento subacromial com ou sem tenotomia ou tenodese da cabeça longa do bíceps $(\mathrm{CLB})^{(6-13)}$, reparo parcial ${ }^{(14,15)}$, transferências miotendíneas $^{(16-18)}$ e substituição protética ${ }^{(19,20)}$.

O desbridamento artroscópico é indicado para aqueles pacientes com ruptura extensa e irreparável, idosos, com baixa demanda funcional e que tenham pré-operatoriamente boa mobilidade do ombro. Além disso, é um procedimento cirúrgico que visa melhorar a dor, porém sem melhora da força.

O objetivo deste trabalho foi avaliar funcionalmente os pacientes submetidos à desbridamento artroscópico das lesões extensas e irreparáveis do manguito rotador, associado ou não à tenotomia da CLB e, em alguns casos, tuberoplastia como preconizado por Fenlin et $a l^{(21)}$.

\section{MATERIAL E MÉTODOS}

Entre 2003 e 2007 foram operados por artroscopia 27 pacientes com ruptura extensa e irreparável do MR, sendo que desses foram avaliados 22 pacientes (sete homens e 15 mulheres) com idade média de 69 anos ( 50 a 84). O lado dominante estava envolvido em 17 pacientes.

Foram excluídos pacientes que apresentavam cirurgia prévia no ombro afetado, sinais clínicos e por imagens de artrose glenoumeral e aqueles que sofreram reparo parcial ou total do MR durante o ato cirúrgico.

Tratamento conservador prévio foi realizado por pelo menos seis meses com fisioterapia, analgésicos e anti-inflamatórios, além de infiltração com corticosteroide, quando indicada. O diagnóstico de ruptura extensa e irreparável foi feito através do exame clínico, por ressonância magnética $(\mathrm{RM})$ e pela incapacidade de reparo dessas lesões durante o ato cirúrgico. Durante o exame clínico, todos pacientes apresentavam sinais de impacto, diminuição da força do supraespinal (SE) e do infraespinal (IE), sem atrofia muscular, porém com hipotrofia da fossa supra e infraescapular em alguns pacientes. Utilizamos os critérios de avaliação da UCLA.

A ressonância magnética foi realizada em todos pacientes no pré-operatório e em 16 pacientes no pósoperatório. Avaliamos o grau de atrofia e degeneração gordurosa, bem como o grau de retração dos tendões, segundo Thomazeau et $a l^{(22)}$, Goutallier et $a l^{(23)} \mathrm{e}$ Patte $^{(2)}$, respectivamente.

A artroscopia seguiu os padrões habituais com visualização pelo portal posterior e instrumentação pelos portais anterior e lateral. Primeiramente, avaliou-se a articulação glenoumeral com o objetivo de identificar alterações labrais ou degenerativas da própria articulação, rupturas do MR e a qualidade da CLB, que foi tenotomizada quando preenchia um dos seguintes critérios: sinovite, lesão maior do que $50 \%$ de sua espessura, subluxação ou luxação. Não foi feita tenodese em nenhum caso.

Já no espaço subacromial seguiu-se com bursectomia, sinovectomia parcial, visualização das lesões, desbridamento da área avascular dos tendões envolvidos e exérese do esporão subacromial quando presente. A acromioplastia não foi feita em nenhum paciente, pois é importante a manutenção do arco coracoacromial para esse tipo de lesão, impedindo ainda mais ascensão da cabeça umeral. Ressecção da clavícula distal também não foi realizada em nenhum caso.

Todos os tendões foram testados previamente quanto à sua redutibilidade e se estavam friáveis, sendo que somente após esse procedimento eram classificados como irreparáveis. Tuberoplastia realizada quando havia sinais de impacto subacromial, exostose na tuberosidade maior e redução importante do espaço subacromial.

As radiografias no pós-operatório em AP verdadeiro e perfil escapular foram para observar presença de artrose glenoumeral e ascensão da cabeça umeral, segundo os critérios de Hamada et al ${ }^{(24)}$. A RM no pósoperatório teve objetivo de avaliar o envolvimento de outros tendões e se houve aumento da atrofia e do grau de degeneração gordurosa.

No pós-operatório imediato, os pacientes eram mantidos em uma tipoia tipo Velpeau para conforto e alívio da dor. Exercícios autopassivos para ombro e cotovelo iniciados com 24 horas após a cirurgia e os pontos retirados com sete dias. O fortalecimento muscular com orientação fisioterápica foi iniciado após duas semanas. Todos pacientes foram submetidos à avaliação subjetiva do grau de satisfação, perguntando se eles estavam muito satisfeitos, satisfeitos, desapontados ou insatisfeitos.

\section{RESULTADOS}

Após um follow-up médio de 27 meses (oito a 60 meses) 22 dos 27 pacientes foram avaliados (Tabelas 1 e 2). Houve melhora dos critérios da UCLA, passando de 
Tabela 1 - Pacientes avaliados.

\begin{tabular}{|c|c|c|c|c|c|c|c|c|c|}
\hline \multirow{2}{*}{ Número } & \multirow{2}{*}{$\begin{array}{l}\text { Tenotomia } \\
\text { do bíceps }\end{array}$} & \multirow{2}{*}{$\begin{array}{c}\text { Força } \\
\text { muscular }\end{array}$} & \multirow{2}{*}{ Satisfação } & \multirow{2}{*}{$\begin{array}{l}\text { Hamada } \\
\text { Pós }\end{array}$} & \multirow{2}{*}{$\begin{array}{c}\text { Ressonância magnética - } \\
\text { Redondo menor }\end{array}$} & \multirow{2}{*}{$\begin{array}{l}\text { Tipo de } \\
\text { acrômio }\end{array}$} & \multicolumn{3}{|c|}{ ADM } \\
\hline & & & & & & & EA & RE & $\mathbf{R I}$ \\
\hline 1 & Sim & $\mathrm{D}$ & M.S. & 1 & Normal & Reto & 180 & 45 & T7 \\
\hline 2 & Não & D & M.S. & 2 & Normal & Curvo & 160 & 60 & T10 \\
\hline 3 & Sim & D & S. & 2 & Normal & Curvo & 150 & 40 & $\mathrm{~T} 11$ \\
\hline 4 & Não & $D$ & M.S. & 1 & Normal & Ganchoso & 180 & 30 & $\mathrm{~T} 10$ \\
\hline 5 & Sim & D & S. & 3 & Normal & Curvo & 130 & 50 & L1 \\
\hline 6 & Não & D & S. & 2 & Discreta degeneração e atrofia & Ganchoso & 170 & 60 & $\mathrm{~T} 10$ \\
\hline 7 & Não & $\mathrm{D}$ & M.S. & 2 & & Ganchoso & 160 & 40 & $\mathrm{~T} 12$ \\
\hline 8 & Não & $\mathrm{D}$ & M.S. & 2 & Normal & Curvo & 150 & 30 & T7 \\
\hline 9 & Sim & $\mathrm{D}$ & M.S. & 2 & Normal & Ganchoso & 170 & 50 & $\mathrm{~T} 7$ \\
\hline 10 & Sim & $\mathrm{D}$ & M.S. & 2 & Normal & Curvo & 120 & 40 & L4 \\
\hline 11 & Sim & $\mathrm{D}$ & S. & 2 & Normal & Curvo & 180 & 90 & T7 \\
\hline 12 & Não & $\mathrm{D}$ & M.S. & 2 & Normal & Curvo & 150 & 60 & $\mathrm{~T} 10$ \\
\hline 13 & Sim & Igual & M.S. & 1 & & Curvo & 180 & 60 & T7 \\
\hline 14 & Não & $D$ & M.S. & 2 & Normal & Ganchoso & 180 & 50 & T7 \\
\hline 15 & Sim & Igual & M.S. & 3 & Normal & Reto & 180 & 60 & $\mathrm{~T} 12$ \\
\hline 16 & Sim & Igual & M.S. & 2 & Atrofia e degeneração gordurosa & Curvo & 180 & 50 & T9 \\
\hline 17 & Não & $\mathrm{D}$ & M.S. & 2 & Normal & Curvo & 180 & 30 & $\mathrm{T7}$ \\
\hline 18 & Sim & $\mathrm{D}$ & $\mathrm{D}$ & 2 & Normal & Curvo & 150 & 50 & L2 \\
\hline 19 & Não & $\mathrm{D}$ & M.S. & 1 & & Reto & 180 & 60 & T7 \\
\hline 20 & Sim & $D$ & M.S. & 2 & & Curvo & 120 & 10 & $\mathrm{~T} 7$ \\
\hline 21 & Sim & $D$ & M.S. & 3 & & Curvo & 180 & 45 & $\mathrm{~T} 7$ \\
\hline 22 & Não & D & M.S. & 1 & & Reto & 180 & 10 & $\mathrm{~T} 7$ \\
\hline
\end{tabular}

15 em média no pré-operatório para 31 no pós, sendo $81,81 \%$ de bons e excelentes resultados, $18,19 \%$ de resultados razoáveis e nenhum resultado ruim.

Não encontramos diferença nos resultados quando avaliamos os pacientes em relação à idade, sexo ou profissão. O lado dominante estava envolvido em 17 pacientes.

Em relação ao grau de satisfação, 21 pacientes estavam satisfeitos/muito satisfeitos e nenhum insatisfeito, porém um paciente estava desapontado. A força muscular estava diminuída em 19 pacientes, mantida em três e em nenhum aumentou.

A rotação externa média foi de $47^{\circ}$ e a elevação anterior média de $164^{\circ}$. Paciente em pós-operatório do ombro direito (Figura 1).

Foram realizadas 12 tenotomias da CLB com presença de deformidade de Popeye em cinco, porém sem queixas dessa deformidade ou de perda de força.

$\mathrm{O}$ tendão do SE estava rompido em todos pacientes e com grau de retração tipo 3, segundo Patte. O tendão do IE também estava envolvido em todos pacientes.
$\mathrm{Na}$ RM antes da cirurgia não identificamos lesões do subescapular e do redondo menor, porém na avaliação pós-operatória por RM identificamos sete tendinopatias e três rupturas parciais do subescapular, além de dois pacientes com leve atrofia e degeneração gordurosa do redondo menor.

Encontramos também degeneração gordurosa nos 16 pacientes avaliados com RM, sendo nove Goutalier IV e sete Goutalier III; além disso, encontramos também atrofia do ventre muscular do SE tipo C de Thomazeau em 10 pacientes e tipo B em seis RM pré-operatório (Figura 2).

As radiografias mostraram, segundo os critérios de Hamada, cinco pacientes com grau 1, 14 com grau 2 e três com grau 3. Não encontramos nenhum grau 4 ou 5.

\section{DISCUSSÃO}

Lesões do MR ocorrem normalmente em pacientes idosos, seja de forma traumática ou atraumática. Geralmente podem ser assintomáticas e não causar muita limitação funcional. Quando sintomáticas, passam a ser um grande problema para esses pacientes, pois interferem 
Tabela 2 - Pacientes avaliados.

\begin{tabular}{|c|c|c|c|c|c|c|c|c|c|}
\hline Número & Idade & Sexo & Ocupação & $\begin{array}{l}\text { Lado afetado/ } \\
\text { Lado dominante }\end{array}$ & $\begin{array}{l}\text { Tendões } \\
\text { envolvidos }\end{array}$ & $\begin{array}{l}\text { Follow-up } \\
\text { (meses) }\end{array}$ & UCLA Pré & UCLA Pós & Popeye \\
\hline 1 & 57 & $\mathrm{M}$ & Aposentado & $\mathrm{D} / \mathrm{D}$ & SE / IE & 8 & 9 & 34 & Sim \\
\hline 2 & 77 & $\mathrm{~F}$ & Aposentado & $D / D$ & SE / IE & 44 & 22 & 32 & Não \\
\hline 3 & 76 & $\mathrm{~F}$ & Aposentado & $\mathrm{D} / \mathrm{D}$ & SE / IE & 44 & 5 & 29 & Não \\
\hline 4 & 50 & $\mathrm{~F}$ & Do lar & $D / D$ & SE / IE & 22 & 15 & 31 & Não \\
\hline 5 & 79 & $\mathrm{~F}$ & Do lar & $\mathrm{D} / \mathrm{D}$ & SE / IE & 51 & 12 & 25 & Sim \\
\hline 6 & 78 & $\mathrm{~F}$ & Aposentado & $\mathrm{D} / \mathrm{D}$ & SE / IE & 58 & 15 & 32 & Não \\
\hline 7 & 65 & $\mathrm{~F}$ & Auxiliar Serv. Gerais & $D / D$ & SE / IE & 24 & 16 & 26 & Não \\
\hline 8 & 66 & $\mathrm{~F}$ & Servente & $D / D$ & SE / IE & 27 & 22 & 30 & Não \\
\hline 9 & 51 & M & Motorista & $\mathrm{D} / \mathrm{D}$ & SE / IE & 11 & 16 & 34 & Sim \\
\hline 10 & 71 & M & Aposentado & $E / D$ & SE / IE & 8 & 12 & 27 & Sim \\
\hline 11 & 63 & $\mathrm{M}$ & Motorista & $E / D$ & SE / IE & 10 & 11 & 34 & Sim \\
\hline 12 & 70 & $\mathrm{~F}$ & Aposentado & $D / D$ & SE / IE & 60 & 10 & 25 & Não \\
\hline 13 & 78 & $\mathrm{~F}$ & Do lar & $E / D$ & SE / IE & 29 & 15 & 35 & Não \\
\hline 14 & 69 & $\mathrm{~F}$ & Costureira & $E / D$ & SE / IE & 35 & 15 & 30 & Não \\
\hline 15 & 60 & $\mathrm{M}$ & Comerciante & $\mathrm{D} / \mathrm{D}$ & SE / IE & 11 & 27 & 35 & Não \\
\hline 16 & 70 & $\mathrm{~F}$ & Do lar & $D / D$ & SE / IE & 8 & 12 & 32 & Não \\
\hline 17 & 80 & $\mathrm{~F}$ & Aposentado & $\mathrm{D} / \mathrm{D}$ & SE / IE & 45 & 17 & 34 & Não \\
\hline 18 & 67 & $\mathrm{M}$ & Aposentado & $E / D$ & SE / IE & 23 & 16 & 28 & Não \\
\hline 19 & 65 & $\mathrm{M}$ & Aposentado & $D / D$ & SE / IE & 27 & 17 & 29 & Não \\
\hline 20 & 80 & $\mathrm{~F}$ & Do lar & $D / D$ & SE / IE & 12 & 8 & 29 & Não \\
\hline 21 & 59 & $\mathrm{~F}$ & Do lar & $D / D$ & SE / IE & 10 & 15 & 34 & Não \\
\hline 22 & 80 & $F$ & Do lar & $D / D$ & SE / IE & 18 & 18 & 34 & Não \\
\hline
\end{tabular}

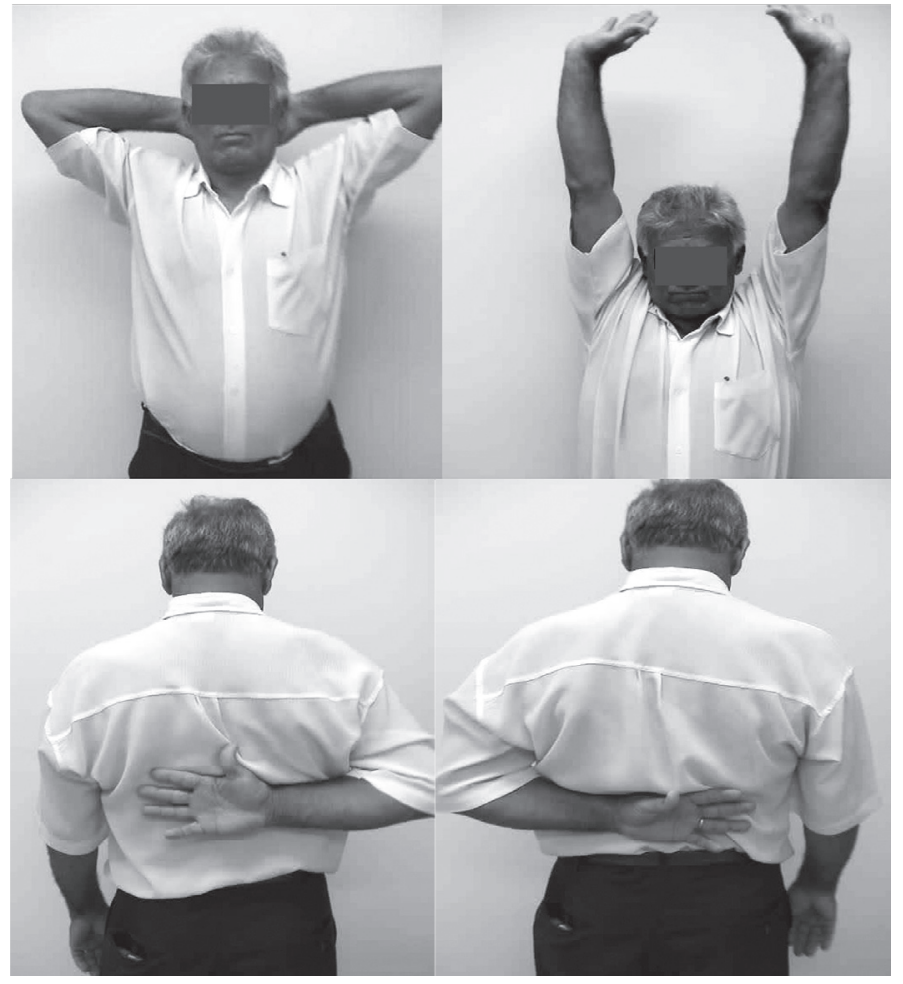

Figura 1 - Paciente em pós-operatório do ombro direito. principalmente no sono e nas atividades da vida diária.

$\mathrm{O}$ tratamento deve ser inicialmente sempre conservador, com controle da dor, exercícios de alongamentos e fortalecimento muscular conforme preconizou Rockwood no seu clássico programa de reabilitação denominado de "ortoterapia"(8).

Especificamente nas lesões extensas, o tratamento conservador com reabilitação e fortalecimento do deltoide anterior deve ser tentado antes de qualquer procedimento cirúrgico, como foi muito bem orientado por Levy et $a l^{(3)}$.

Quando ocorre falha desse tratamento passa-se a ter indicação para o tratamento cirúrgico. Especificamente nessa faixa etária, temos algumas peculiaridades em relação à ruptura do MR e aos próprios pacientes: geralmente são rupturas crônicas, com grande retração tendínea, com tecido muito friável, com presença de atrofia muscular e substituição gordurosa, que geralmente levam à não consolidação dessas lesões.

Além disso, como são pacientes de baixa demanda funcional, os bons resultados cirúrgicos passam 


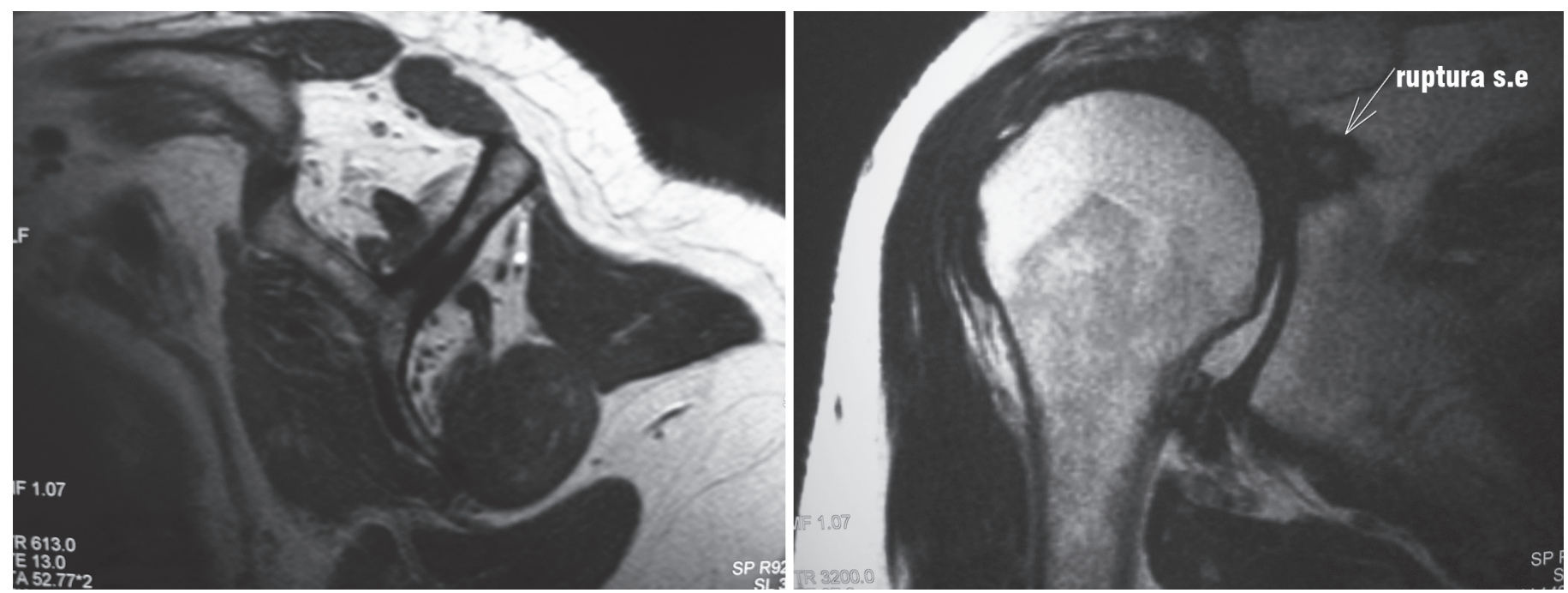

Figura 2 - Corte sagital e coronal da ressonância magnética mostrando atrofia do SE com infiltração gordurosa, ruptura completa com retração no nível da glenoide e ascensão da cabeça umeral.

principalmente pelo alívio da dor. Sendo assim, o desbridamento artroscópico torna-se boa opção cirúrgica para esses pacientes principalmente com esse padrão de ruptura, pois é um procedimento rápido, de baixa morbidade, que não desinsere o deltoide, com baixos índices de infecção e que permite início precoce da reabilitação.

Nossos resultados clínicos mostraram que os pacientes apresentaram boa resposta ao tratamento, sendo que houve melhora significativa dos critérios da UCLA. Somente um paciente estava desapontado com o resultado cirúrgico, pois não houve melhora do quadro álgico e também não apresentou uma boa amplitude de movimento (ADM).

Observamos um dado importante, que também está de acordo com a literatura, exceto pelo trabalho de Liem et $a l^{(25)}$, que foi a não recuperação da força muscular em 19 pacientes. Era um resultado esperado em função do padrão das lesões e da idade dos pacientes, tanto que todos foram informados antes da cirurgia que isso poderia acontecer.

Diante da baixa demanda funcional desses pacientes, consideramos os resultados satisfatórios, pois o que foi proposto pelo tratamento foi conseguido, ou seja, houve melhora de todos os parâmetros analisados. Concordamos com alguns autores, quando eles dizem não saber por quanto tempo esses bons resultados iriam se manter.

Por outro lado, pudemos observar no follow-up que tivemos pacientes sendo acompanhados por 60 meses, sem deterioração do quadro clínico. Zvijac et al ${ }^{(7)}$ mostraram em seu trabalho que houve piora clínica com o passar dos anos. É uma questão que ainda merece ser analisada e discutida.

Foram realizadas 12 tenotomias da CLB, segundo os critérios descritos anteriormente. Observamos presen- ça de deformidade tipo Popeye em menos de 50\% dos casos. Isso confirma que mesmo diante de uma lesão extensa pode ocorrer auto tenodese da CLB na goteira bicipital, desde que se tenha o ligamento transverso umeral íntegro, pois normalmente a goteira é mais estreita do que a porção intra-articular do tendão.

Não observamos clinicamente alterações da força de supinação e flexão do cotovelo desses pacientes tenotomizados, quando comparados com aqueles sem tenotomia. Além disso, também não houve queixa com relação à estética daqueles pacientes que apresentaram deformidade tipo Popeye, talvez em função da própria idade e de outros objetivos de vida, que não a cosmética.

Tivemos muito cuidado em comparar a RM antes e após a cirurgia, principalmente em relação aos tendões envolvidos. Achamos que talvez nossa amostra estivesse viciada, já que todos os pacientes apresentavam no pré-operatório apenas lesão do SE e do IE. Porém, ao confrontar nossa casuística com a casuística de Fenlin et $a l^{(21)}$, observamos que eles também apresentaram somente lesão do SE e do IE em 18 dos 19 pacientes avaliados pela IRM.

Por outro lado, isso nos permitiu dar ênfase à avaliação do redondo menor, pois concordamos com a hipótese descrita por Burkhart ${ }^{(26)}$, que desde que tenhamos o MR anterior (subescapular) e o posterior (IE e redondo menor) contrabalanceados, podemos ter uma "lesão anatomicamente deficiente, porém intacta biomecanicamente".

Como em todos pacientes avaliados o IE estava lesado, tivemos como rotador externo apenas o redondo menor e mesmo assim observamos uma rotação externa média de $47^{\circ}$ no pós-operatório. 
Sendo assim, a teoria de Burkhart foi condizente com os bons resultados, pois tratamos as lesões extensas e suas respectivas causas de dor (sinovite, bordas instáveis do MR, bursite, tendinite bicipital, corpos livres) somente com o desbridamento artroscópico e aproveitamos a estabilidade biomecânica da lesão para fazer uma boa reabilitação, com um programa bem orientado e direcionado para o fortalecimento do deltoide.

Realizamos as radiografias no pós-operatório para avaliarmos se os pacientes evoluíram com artropatia do MR e posteriormente com artrose da articulação glenoumeral, já que essa possibilidade é bem possível de ocorrer, pois os tendões estavam rotos e a articulação teoricamente instável.

Nenhum paciente evoluiu com artrose ou necrose da cabeça umeral, que são respectivamente os graus 4 e 5 de Hamada, sendo que, no máximo, observamos Hamada tipo 3.

Essa observação serve para confirmar novamente que desde que tenhamos ombros biomecanicamente estáveis e mesmo diante de lesões extensas, não há sobrecarga ou prejuízo articular.

A tuberoplastia, segundo Fenlin, não foi realizada em todos pacientes. A melhor indicação para esse procedimento é naqueles pacientes que apresentam o espaço subacromial muito reduzido pela ascensão da cabeça umeral e que se queira criar uma congruência entre o acrômio e a tuberosidade maior. Sendo assim, realizamos a tuberoplastia em apenas dois, dos três pacientes que eram Hamada tipo 3.

\section{CONCLUSÃO}

Em conclusão, os pacientes idosos, com baixa demanda funcional, com queixa principal de dor, que não responderam ao tratamento conservador, com ruptura extensa e irreparável do MR e que apresentam um ombro biomecanicamente estável são bons candidatos ao desbridamento artroscópico, sendo necessário posteriormente avaliarmos se a boa resposta imediata será mantida durante um longo período.

\section{REFERÊNCIAS}

1. DeOrio JK, Cofield RH. Results of a second attempt at surgical repair of a failed initial rotator-cuff repair. J Bone Joint Surg Am. 1984;66(4):563-7.

2. Patte D. Classification of rotator cuff lesions. Clin Orthop Relat Res 1990;(254):81-6.

3. Levy $\mathrm{O}$, Mullett $\mathrm{H}$, Roberts $\mathrm{S}$, Copeland $\mathrm{S}$. The role of anterior deltoid reeducation in patients with massive irreparable degenerative rotator cuff tears. $J$ Shoulder Elbow Surg. 2008;17(6):863-70.

4. Lech O, Valenzuela NC, Severo A. Tratamento conservador das lesões parciais e completas do manguito rotador. Acta Ortop Bras. 2000;8(3):144-56.

5. Zingg PO, Jost B, Sukthankar A, Buhler M, Pfirrmann CW, Gerber C. Clinical and structural outcomes of nonoperative management of massive rotator cuff tears. J Bone Joint Surg Am. 2007;89(9):1928-34

6. Burkhart SS. Arthroscopic treatment of massive rotator cuff tears. Clinical results and biomechanical rationale. Clin Orthop Relat Res. 1991 Jun;(267):45-56.

7. Zvijac JE, Levy HJ, Lemak LJ. Arthroscopic subacromial decompression in the treatment of full thickness rotator cuff tears: a 3- to 6-year follow-up. Arthroscopy. 1994;10(5):518-23.

8. Rockwood CA Jr, Williams GR Jr, Burkhead WZ Jr. Débridement of degenerative irreparable lesions of the rotator cuff. J Bone Joint Surg Am. 1995;77(6):857-66

9. Levy HJ, Gardner RD, Lemak LJ. Arthroscopic subacromial decompression in the treatment of full-thickness rotator cuff tears. Arthroscopy. 1991;7(1):8-13.

10. Scheibel M, Lichtenberg S, Habermeyer P. Reversed arthroscopic subacromial decompression for massive rotator cuff tears. J Shoulder Elbow Surg. 2004;13(3):272-8

11. Boileau P, Baqué F, Valerio L, Ahrens P, Chuinard C, Trojani C. Isolated arthroscopic biceps tenotomy or tenodesis improves symptoms in patients with massive irreparable rotator cuff tears. J Bone Joint Surg Am. 2007;89(4):747-57.

12. Checchia SL, Doneux PS, Miyazaki AN, Fregoneze M, Silva LA, Oliveira FM, et al. Tenotomia artroscópica do bíceps nas lesões irreparáveis do manguito rotador. Rev Bras Ortop. 2003;38(9):513-21.

13. Zoppi AF, Kikuta FK, Pereira LAR, Zan RA. Treatment of irreparable rotator cuff tears by arthroscopy dèbridement. In: 10th International Congress of Shoulder and Elbow Surgery. Brazil, Setembro, 2007.

14. Burkhart SS, Nottage WM, Ogilvie-Harris DJ, Kohn HS, Pachelli A. Partial repair of irreparable rotator cuff tears. Arthroscopy. 1994;10(4):363-70.

15. Duralde XA, Bair B. Massive rotator cuff tears: the result of partial rotator cuff repair. J Shoulder Elbow Surg. 2005;14(2):121-7.

16. Warner JJ. Management of massive irreparable rotator cuff tears: the role of tendon transfer. Instr Course Lect. 2001;50:63-71.

17. Iannotti JP, Hennigan S, Herzog R, Kella S, Kelley M, Leggin B, et al. Latissimus dorsi tendon transfer for irreparable posterosuperior rotator cuff tears. Factors affecting outcome. J Bone Joint Surg Am. 2006;88(2):342-8.

18. Gerber C, Maquieira G, Espinosa N. Latissimus dorsi transfer for the treatment of irreparable rotator cuff tears. J Bone Joint Surg Am. 2006;88(1):113-20.

19. Frankle M, Levy JC, Pupello D, Siegal S, Saleem A, Mighell M, et al. The reverse shoulder prosthesis for glenohumeral arthritis associated with severe rotator cuff deficiency. a minimum two-year follow-up study of sixty patients surgical technique. J Bone Joint Surg Am. 2006;88(Suppl 1 Pt 2):178-90.

20. Wall B, Nové-Josserand L, O'Connor DP, Edwards TB, Walch G. Reverse total shoulder arthroplasty: a review of results according to etiology. J Bone Joint Surg Am. 2007;89(7):1476-85.

21. Fenlin JM Jr, Chase JM, Rushton SA, Frieman BG. Tuberoplasty: creation of an acromiohumeral articulation-a treatment option for massive, irreparable rotator cuff tears. J Shoulder Elbow Surg. 2002;11(2):136-42.

22. Thomazeau H, Boukobza E, Morcet N, Chaperon J, Langlais F. Prediction of rotator cuff repair results by magnetic resonance imaging. Clin Orthop Relat Res. 1997;(344):275-83.

23. Goutallier D, Postel JM, Bernageau J, Lavau L, Voisin MC. Fatty muscle degeneration in cuff ruptures. Pre- and postoperative evaluation by CT scan. Clin Orthop Relat Res. 1994;(304):78-83.

24. Hamada K, Fukuda H, Mikasa M, Kobayashi Y. Roentgenographic findings in massive rotator cuff tears. A long-term observation. Clin Orthop Relat Res. 1990;(254):92-6.

25. Liem D, Lengers N, Dedy N, Poetzl W, Steinbeck J, Marquardt B. Arthroscopic debridement of massive irreparable rotator cuff tears. Arthroscopy. 2008;24(7):743-8.

26. Burkhart SS. Reconciling the paradox of rotator cuff repair versus debridement: a unified biomechanical rationale for the treatment of rotator cuff tears. Arthroscopy. 1994;10(1):4-19. 